\title{
Ferroelectricity and Phase Transitions in Pyridinium Periodate Single Crystal
}

\author{
Z. Czapla, S. Dacko, and B. Kosturek \\ Institute of Experimental Physics, Wrocław University, M. Borna 9, 50-204 Wrocław, Poland \\ Reprint requests to Prof. Z. C.; E-mail: czapla@ferroic.ifd.uni.wroc.pl \\ Z. Naturforsch. 55 a, 891-894 (2000); received August 8, 2000
}

\begin{abstract}
Single crystals of pyridinium periodate were grown, and their physical properties were studied. Anomalies of the electric permittivity and birefringence were observed at $T_{\mathrm{cl}}=321 \mathrm{~K}$ and $T_{\mathrm{c} 2}=$ $211 \mathrm{~K}$. These observations gave evidences for the existence of three phases denoted as I, II, and III. Hysteresis loops were observed both in phase II and III. Pyroelectric measurements showed two anomalies at $T_{\mathrm{cl}}$ and $T_{\mathrm{c} 2}$. The anomaly at $T_{\mathrm{c} 1}$ is related to the transition between a para- and ferroelectric phase, and the anomaly at $T_{\mathrm{c} 2}$ to the transition between two ferroelectric phases.
\end{abstract}

Key words: Phase Transitions; Ferroelecticity; Dielectric Properties; Birefringence.

\section{Introduction}

Pyridinium salts exhibit structural phase transitions [1 - 4]. Pyridinium tetrafluoroborate [5] and perchlorate [6] single crystals were found to exhibit two successive phase transitions and ferroelectricity in the low temperature phases. Studies of powdered pyridinium perrhenate [7] and periodate [8] showed two successive phase transitions $\left(T_{\mathrm{cl}}=333 \mathrm{~K}, T_{\mathrm{c} 2}=\right.$ $250 \mathrm{~K}$ for pyridinium perrhenate and $T_{\mathrm{cl}}=323 \mathrm{~K}$, $T_{\mathrm{c} 2}=206 \mathrm{~K}$ for pyridinium periodate), the three phases being numbered as I, II, and III for descending temperature. Hysteresis loops and ferroelectric properties were observed for the room temperature phase II [8]. The molecular mechanism of the phase transitions is not fully explained, alignment of the non-centrosymmetric pyridinium cations being assumed to cause the spontaneous polarization [9]. There exist no single crystal studies on these two crystals. Therefore we decided to grow single crystals of pyridinium periodate and investigate their dielectric, ferroelectric, pyroelectric, and optical properties and the character of their phase transitions. At room temperature, phase II of pyridinium periodate crystallizes in the orthorhombic system with space group $\mathrm{Cmc2}_{1}$ [9], and the ferroelectric properties are expected to appear along the crystallographic $c$-axis.

\section{Experimental}

Single crystals of pyridinium periodate were grown from saturated water solution of pyridinium and periodate acid at $305 \mathrm{~K}$. Samples of the size $3 \times 4 \times 1 \mathrm{~mm}^{3}$, oriented along the three crystallographic axes, were prepared and used for the studies. The dielectric properties were investigated by means of an HP 4284 A LCR meter at $1 \mathrm{kHz}$ and a measuring field amplitude of $1.5 \mathrm{~V} / \mathrm{cm}$. Hysteresis loops were observed using a modified Sawyer-Tower circuit at of $10^{-2} \mathrm{~Hz}$. The temperature dependence of the spontaneous polarisation was estimated from pyroelectric measurements. Linear birefringence changes were measured using the rotating-analyser method [10]. A $1 \mathrm{~mW}$ He-Ne laser $(\lambda=632.8 \mathrm{~nm})$ was used as light source. The measurements were done with an accuracy of $10^{-6}$. Absolute values of birefringences were determined at room temperature, using of an Ehringhaus compensator with an accuracy of $5 \%$. All temperature dependences were observed at $350-180 \mathrm{~K}$ on cooling/heating runs with a rate of $0.5 \mathrm{~K} / \mathrm{min}$ and $0.05 \mathrm{~K} / \mathrm{min}$ in the phase transition region.

\section{Results and Conclusions}

It has been proposed to obtain pyridinium periodate by neutralization of pyridinium in methanol solution 


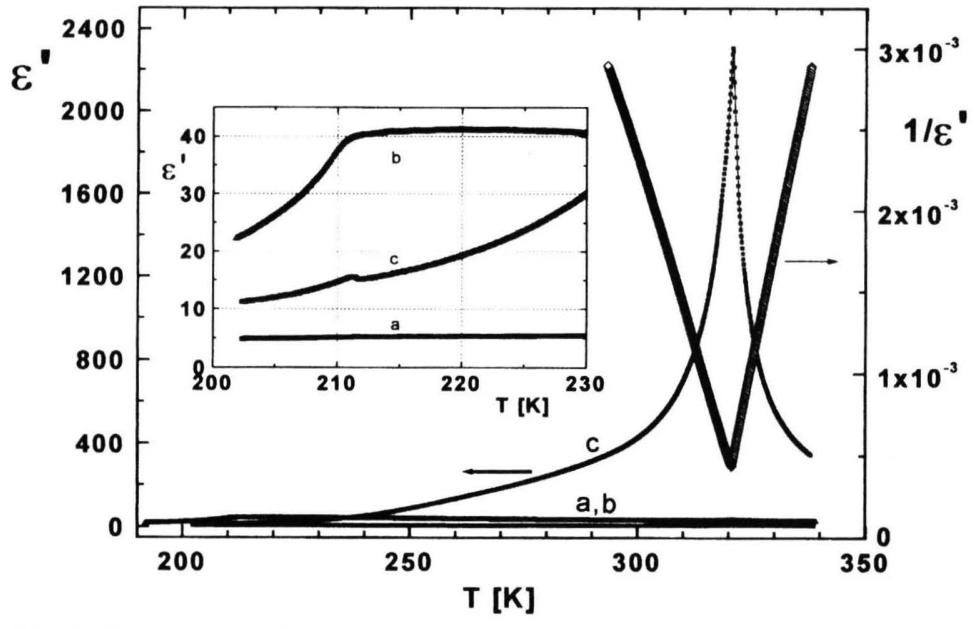

Fig. 1. Temperature dependences of electric permittivity along the $a$-, $b$-, $c$-axes and the reciprocal permittivity along the $c$-axis for pyridinium periodate crystal. The inset represents the electric permittivity in the vicinity of the lower transition.

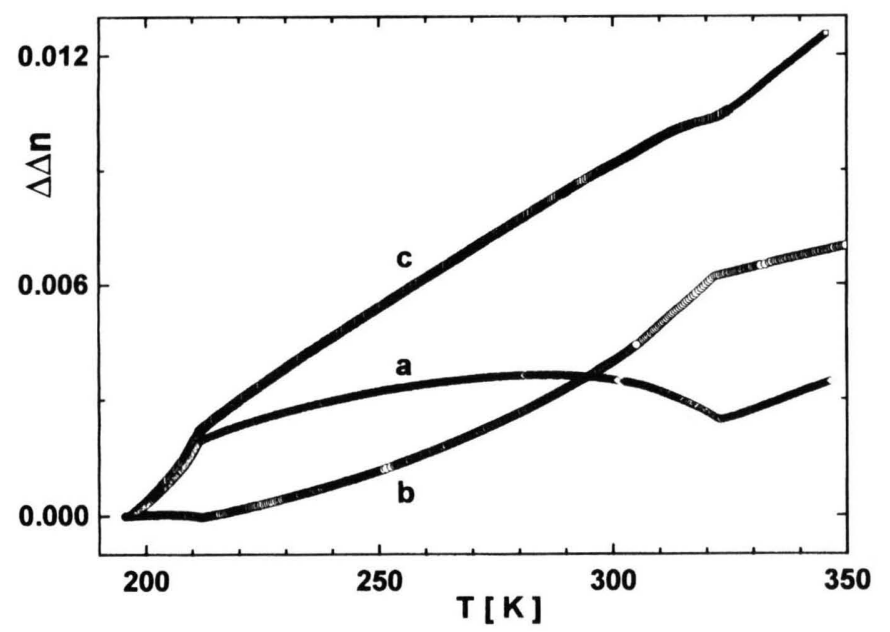

Fig. 3. Temperature dependences of the birefringence changes along the $a-, b-, c$-axes for pyridinium periodate crystal.

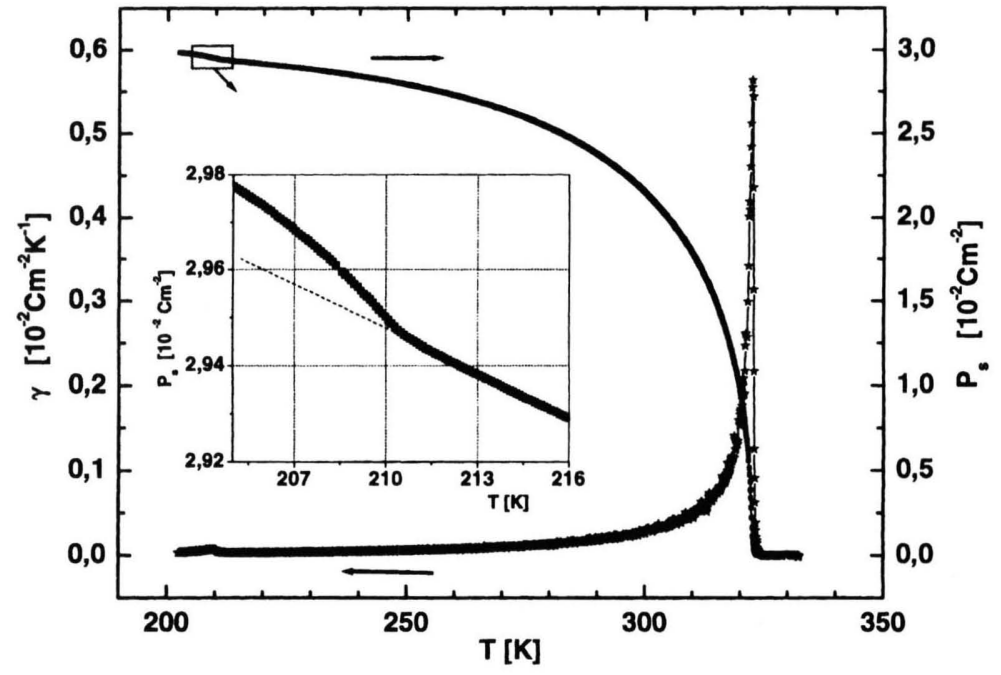

Fig. 2. Temperature dependence of the pyroelectric coefficient $(\gamma)$ and spontaneous polarisation $\left(P_{\mathrm{s}}\right)$ along the $c$-axis of pyridinium periodate crystal; an inset represents the changes of spontaneous polarisation in the vicinity of the lower phase transition.

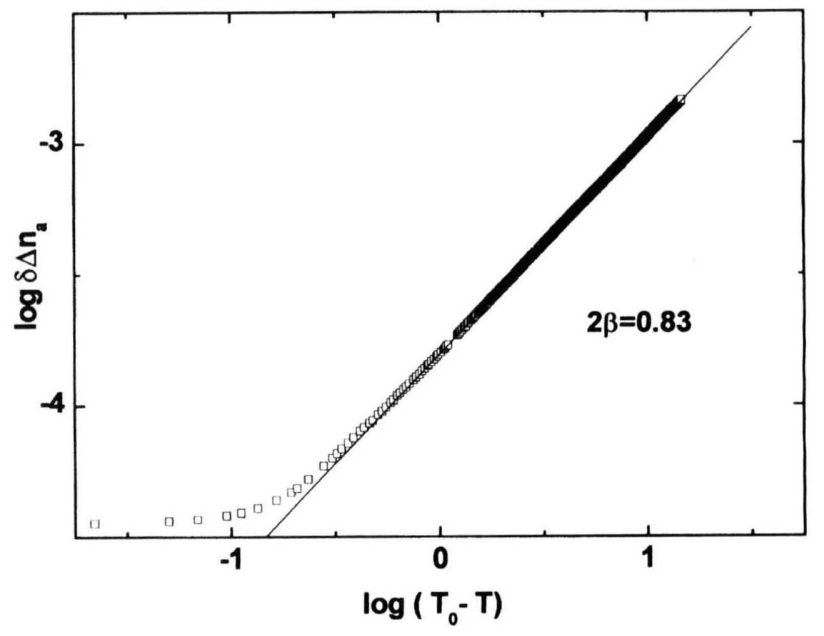

Fig. 4. Log- $\log$ plot of the $\delta \Delta n_{a}$ vs. $\left(T_{\mathrm{c} 2}-T\right)$ for pyridinium periodate crystal. 
with periodic acid and to grow single crystals for Xray structural investigations by slow evaporation of an ethanol solution of the salt [10]. We succeeded to obtain this substance and grow single crystals from saturated water solution. It is difficult to obtain large and good optical quality single crystals of this compound because of its relatively low solubility. We obtained crystals with a volume of $0.8 \mathrm{~cm}^{3}$. The orthorhombic symmetry of the crystals was evident from the crystal habit. Samples of suitable size for measurements were cut out from good optical quality parts of the grown crystals.

Temperature dependences of the electric permittivity and fulfilment of the Curie-Weiss law are shown in Figure 1.

The hysteresis loop measurement at $297.7 \mathrm{~K}$ gives a spontaneous polarization of $2.24 \cdot 10^{-3} \mathrm{C} / \mathrm{m}^{2}$ in comparison to $0.1 \cdot 10^{-3} \mathrm{C} / \mathrm{m}^{2}$ found for pellet samples [8]. Hysteresis loops with the frequency $10^{-2} \mathrm{~Hz}$ could be observed down to $200 \mathrm{~K}$ in spite of the large coercive field. This means that hysteresis loops were also observed below $T_{\mathrm{c} 2}$. These observations give evidence that the phases II and III are ferroelectric. To find the temperature dependence of spontaneous polarization, pyroelectric properties of the crystal were investigated. Temperature dependences of the pyroelectric coefficient and spontaneous polarization (obtained by integration of the pyroelectric coefficient) are shown in Figure 2.

The pyroelectric measurements showed an anomaly at $T_{\mathrm{cl}}$ connected with the appearance of the ferroelectric phase. An increase of spontaneous polarization is observed down to $T_{\mathrm{c} 2}$ where an additional anomalous increase of the spontaneous polarization is observed. The inset in Fig. 2 shows the small anomalous change of polarization connected with the II-III phase transition. An appearance of spontaneous polarization at $T_{\mathrm{c} 1}$ and its changes at $T_{\mathrm{c} 2}$ are characteristic for continuous phase transitions.

Microscopic observations did not reveal any changes of the optical indicatrix position in the range of $350-200 \mathrm{~K}$. This means that orthorhombic symmetry is preserved in all known phases of the crystal. The room temperature birefringences obtained by the measurements are $\Delta n_{a}=-0.07, \Delta n_{b}=0.07$, and
$\Delta n_{c}=0.14$. Results of linear birefringence change measurement as a functions of temperature along three crystallographic directions are shown in Figure 3.

Anomalous changes of birefringence are clearly seen both at $T_{\mathrm{c} 1}$ and $T_{\mathrm{c} 2}$. To describe the character of the phase transitions, the spontaneous contribution to birefrigence connected with the phase transitions (birefringence increment: $\delta \Delta n_{i}$ ) were obtained by subtraction of the birefringence extrapolated from higher to lower phases.

According to theoretical considerations, the birefringence increment is proportional to the square of the order parameter (spontaneous polarization) and its temperature change is related to the order parameter as follows:

$$
\delta \Delta n_{i} \sim\left(T_{\mathrm{c}}-T\right)^{2 \beta} .
$$

This relation in log-log scale gives the $2 \beta$ parameter and is illustrated for the $a$-direction of lower phase transition in Figure 4.

The procedure was applied to the three crystallographic directions and both phase transitions. The $\beta$ parameter (critical exponent) ranges from 0.45 to 0.49 for the para-ferroelectric phase transition $\left(T_{\mathrm{cl}}\right)$ and 0.40 to 0.46 for the ferro-ferroelectric phase transition $\left(T_{\mathrm{c} 2}\right)$. These parameters are close to the values predicted for continuous phase transitions.

Our studies of pyridinium iodate single crystal can be summarised as follows:

1. two succesive continuous phase transitions were confirmed at $T_{\mathrm{c} 1}=321 \mathrm{~K}$ and $T_{\mathrm{c} 2}=211 \mathrm{~K}$;

2 . the anomaly of the electric permittivity observed at $T_{\mathrm{cl}}$ along the $c$-axis is characteristic for ferroelectric phase transitions;

3. the Curie-Weiss law is fulfilled in a broad temperature range above $T_{\mathrm{cl}}$, and $C=3172 \mathrm{~K}$ is typical for transition of order-disorder type;

4. the hysteresis loops observed below $T_{\mathrm{c} 1}$ and $T_{\mathrm{c} 2}$ prove ferroelectric properties of the phases II and III;

5. orthorhombic symmetry is preserved in all phases of the crystal.

This work was supported by the University of Wrocław under grant 2016/W/IFD/99. 
[1] A. Kozak, M. Grottel, J. Wasicki, and Z. Pajak, Phys. Stat. Sol. (a) 143, 65 (1994).

[2] J. Ripmeester, Can. J. Chem. 54, 3553 (1976).

[3] J. Wạsicki, A. Kozak, Z. Pająak, P. Czarnecki, A. V. Belushkin, and M. A. Adams, J. Chem. Phys. 105, 9470 (1996).

[4] A. Kozak, J. Wa̧sicki, and Z. Pająk, Phase Transitions 57, 153 (1996).

[5] P. Czarnecki, W. Nawrocik, Z. Pająk, and J. Wa̧sicki, Phys. Rev. B49, 1511 (1994).
[6] P. Czarnecki, W. Nawrocik, Z. Pająk, and J. Wa̧sicki, J. Phys.: Condens. Matter 6, 4955 (1994).

[7] J. Wąsicki, P. Czarnecki, Z. Pająk, W. Nawrocik, and W. Szczepański, J. Chem. Phys. 107, 576 (1997).

[8] Z. Pająk, P. Czarnecki, J. Wạsicki, and W. Nawrocik, J. Chem. Phys. 109, 6420 (1998).

[9] G. Dutkiewicz and Z. Pająk, Z. Naturforsch. 53b, 1323 (1998).

[10] J. G. Wood and A. M. Glazer, J. Appl. Cryst. 13, 207 (1979). 